\title{
Pulse wake-up and breakdown investigation of ferroelectric yttrium doped $\mathrm{HfO}_{2}$
}

S. Starschich, S. Menzel, and U. Böttger

Citation: Journal of Applied Physics 121, 154102 (2017); doi: 10.1063/1.4981893

View online: https://doi.org/10.1063/1.4981893

View Table of Contents: http://aip.scitation.org/toc/jap/121/15

Published by the American Institute of Physics

\section{Articles you may be interested in}

Enhancing ferroelectricity in dopant-free hafnium oxide

Applied Physics Letters 110, 022903 (2017); 10.1063/1.4973928

Ferroelectricity in hafnium oxide thin films

Applied Physics Letters 99, 102903 (2011); 10.1063/1.3634052

Understanding ferroelectric Al: $\mathrm{HfO}_{2}$ thin films with Si-based electrodes for 3D applications Journal of Applied Physics 121, 204103 (2017); 10.1063/1.4984068

Ferroelectric and piezoelectric properties of $\mathrm{Hf}_{1-x} \mathrm{Zr}_{x} \mathrm{O}_{2}$ and pure $\mathrm{ZrO}_{2}$ films Applied Physics Letters 110, 182905 (2017); 10.1063/1.4983031

Evidence for oxygen vacancies movement during wake-up in ferroelectric hafnium oxide Applied Physics Letters 108, 032903 (2016); 10.1063/1.4940370

Stabilizing the ferroelectric phase in doped hafnium oxide Journal of Applied Physics 118, 072006 (2015); 10.1063/1.4927805

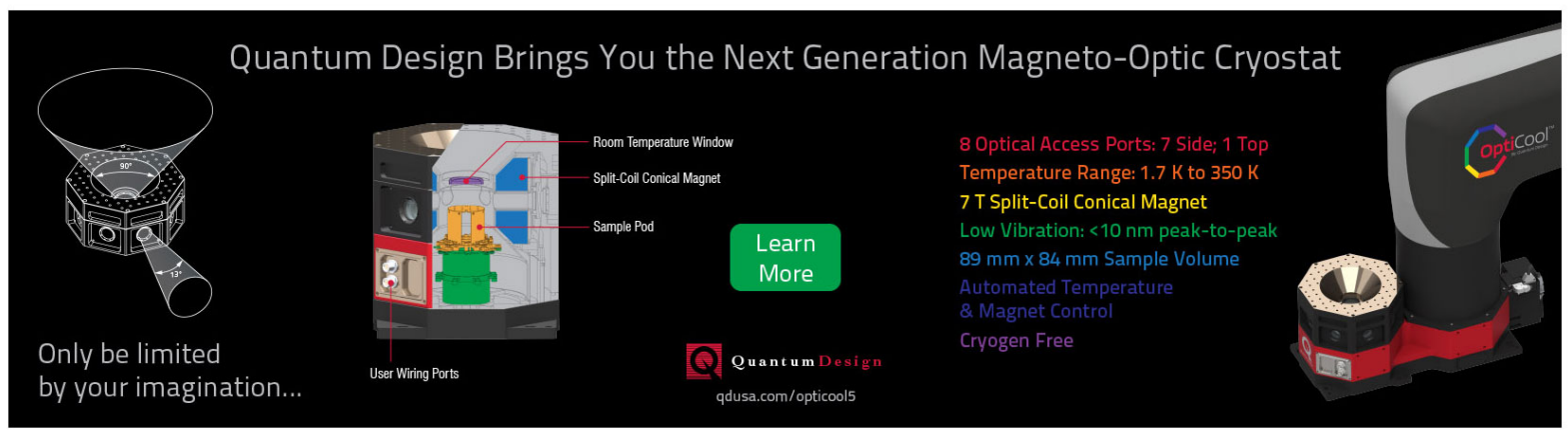




\title{
Pulse wake-up and breakdown investigation of ferroelectric yttrium doped $\mathrm{HfO}_{2}$
}

\author{
S. Starschich, ${ }^{1}$ S. Menzel, ${ }^{2}$ and U. Böttger ${ }^{1}$ \\ ${ }_{1}^{1}$ Institut für Werkstoffe der Elektrotechnik 2, RWTH Aachen University, Sommerfeldstraße 24, \\ D-52074 Aachen, Germany \\ ${ }^{2}$ Peter Grünberg Institut 7, Forschungszentrum Jülich GmbH, D-52425 Jülich, Germany
}

(Received 17 February 2017; accepted 9 April 2017; published online 21 April 2017)

\begin{abstract}
The wake-up effect in yttrium doped hafnium oxide is investigated by pulse measurements, revealing the initial distribution of oxygen vacancies within symmetrical and asymmetrical layer stacks. It is shown that single pulses are sufficient to achieve a significant wake-up, whereby additional pulses lead to a stronger wake-up. Further cycling induces a degradation of the devices, which is explained by generation of oxygen vacancies and results in an electroforming step where subsequently resistive valence change mechanism switching is observed. The degradation and therefore the generation of oxygen vacancies show a strong frequency dependence, whereby the device lifetime is strongly increased for increasing frequencies. Published by AIP Publishing.

[http://dx.doi.org/10.1063/1.4981893]
\end{abstract}

\section{INTRODUCTION}

The 2011 discovered unconventional ferroelectric properties of doped hafnium oxide thin films ${ }^{1}$ enable the application in future low power and high-density nonvolatile memory devices. ${ }^{2-4}$ The ferroelectricity is attributed to the non-centrosymmetric orthorhombic phase with a Pca2 $2_{1}$ space group, which was assumed by several groups ${ }^{1,5-7}$ and was recently proven by Sang et al. ${ }^{5}$

The wake-up effect is attributed to an increase of the remanent polarization during cycling, where mostly a constricted hysteresis is observed in the pristine state, which opens up during cycling. The wake-up was observed in hafnium oxide with different dopants $\left(\mathrm{Si}^{8}, \mathrm{Gd}^{8}, \mathrm{Sr}^{10}\right)$ as well as for the $\mathrm{Hf}_{1-\mathrm{x}} \mathrm{Zr}_{\mathrm{x}} \mathrm{O}_{2}$ system. ${ }^{11}$ Furthermore, for different deposition techniques as sputtering, ${ }^{12}$ chemical solution deposition $^{13}$ and pulsed layer deposition, ${ }^{14}$ initial cycling was also necessary to exhibit the maximum remanent polarization. Therefore, it can be concluded that the wake-up is an intrinsic property of the hafnia and zirconia based ferroelectrics. Understanding the wake-up and degradation mechanism is crucial for increasing the endurance of ferroelectric devices. Several groups discussed the origin of the wake-up. ${ }^{8,15-17}$ Schenk et al. showed two distinct maxima in the $\mathrm{I}(\mathrm{V})$ curve in the pristine state, which are biased against each other and merge together during cycling. ${ }^{16}$ They further assumed that this internal bias could be attributed to mobile ions that are rearranged after wake-up cycling. Recently, it was shown that during the wake-up a redistribution of the oxygen vacancies occurs, leading to a decrease of the built-in bias field and a stabilization of the ferroelectric phase. ${ }^{18,19}$ This redistribution is accompanied by a phase transition, which was assumed and shown by several groups. Park et al. assumed a phase transition during wake-up from a tetragonal to the ferroelectric orthorhombic phase as the origin of the observed wake-up for $8 \mathrm{Hf}_{0.5} \mathrm{Zr}_{0.5} \mathrm{O}_{2}{ }^{15}$ A phase transition from monoclinic to the ferroelectric orthorhombic phase was found by TEM measurements by Grimley et al. and Martin et al. ${ }^{6,20}$
Grimley et al. further considered that the redistribution of oxygen vacancies within the layer leads to a reduction of the barrier for the transformation from the tetragonal to the orthorhombic phase. ${ }^{20}$ Furthermore, Pešic et al. showed that during the degradation (fatigue) of doped hafnium oxide oxygen vacancies are generated, whereas the generation of the vacancies has no influence during the wake-up cycling. The wake-up was explained by redistribution of oxygen vacancies, which are initially accumulated at the interface regions leading to the built-in field bias. ${ }^{19} \mathrm{~A}$ built-in field bias was also recently found in iron doped lead zirconate titanate (PZT) as reported by Fengler et al. ${ }^{21}$ Therefore, the observed wake-up is not only present in hafnia and zirconia based ferroelectrics but also can be found in conventional ferroelectrics.

Besides ferroelectric switching, bipolar resistive switching in hafnium oxide films has been frequently reported. ${ }^{22-28}$ Therefore, the resistance is changed between a high and a low resistance state by applying a bipolar voltage scheme. The resistive switching effect is based on the movement of oxygen vacancies leading to a valence change of the hafnium cations and a change in the local conductivity. ${ }^{29-31}$ Therefore, this mechanism is also referred to as valence change mechanism (VCM). ${ }^{32}$ In general, an electroforming step is required before stable resistive switching is observed. During the electroforming step, oxygen is extracted via one of the electrodes and an oxygen deficient filament is formed. ${ }^{32-34}$ Therefore, in most cases, asymmetrical electrodes are used with an inert electrode on the one hand and an oxygen-scavenging electrode on the other hand. A forming free-device can be achieved by depositing a substoichiometric oxide layer. ${ }^{35}$

In this work, we investigate the ferroelectric wake-up by pulse measurements for symmetrical and asymmetrical electrodes. From these results, the initial oxygen vacancy distribution can be extracted. In the next step, the degradation and breakdown of the devices is evaluated in dependence of the 
applied cycling frequency. Understanding the degradation process of ferroelectric devices is essential for nonvolatile memory application.

\section{EXPERIMENTAL PROCEDURE}

The deposition of the doped hafnium oxide layer is carried out by chemical solution deposition, as described in detail elsewhere. ${ }^{36}$ The platinum electrodes as well as the titanium nitride (TiN) layer are deposited by sputter deposition from a six inch platinum and titanium target, respectively. For the titanium nitride, $10 \%$ nitrogen was added to the argon atmosphere to realize reactive sputtering. The top electrodes were deposited by a negative lift off process, and for the electrical measurements an electrode area of $625 \mu \mathrm{m}^{2}$ was used. The hysteresis measurements were carried out using a Keithley 4200 at a frequency of $1 \mathrm{kHz}$, whereas a Keithley 2410 was used for recording the resistive switching curves. Therefore, the potential was applied to the top electrode, while the bottom electrode was shortened.

\section{RESULTS AND DISCUSSION}

The two layer stacks used in this work are depicted in Fig. 1(a). For both devices, 5\% yttrium doped $\mathrm{HfO}_{2}$ with a thickness of $15 \mathrm{~nm}$ is used between platinum electrodes, whereas for the second device a $20 \mathrm{~nm}$ thick titanium nitride (TiN) layer is sandwiched between the hafnium oxide and the platinum top electrode in order to realize an asymmetric stack structure. TiN was chosen as the oxygen scavenging layer because for other electrodes like, e.g., titanium a significantly lower forming voltage was observed compared to TiN (data not shown here). This did lead to a forming step during wake-up cycling and, therefore, smaller voltage was needed whereby a significantly smaller remanent polarization was observed. The hysteresis curves after a regular wake-up for the symmetrical and asymmetrical stack are shown in Fig. 1(b). A slightly lower coercitive field is found for the asymmetrical stack, whereby for higher electrical fields the polarization is identical for both stacks. Fig. 1(c) shows the current curves for a regular wake-up for the asymmetrical device. During the initial cycle, only weak current peaks can be found. This is consistent with the structural properties of our CSD deposited layers, which were discussed in the previous studies. ${ }^{13,36,37}$ In those studies, it was shown by Gi-XRD that the cubic phase is dominant in the pristine layers and a phase transition takes place during cycling. This corresponds to the electrical results where no ferroelectric switching could be found initially. With ongoing cycling, the initial double peaks merge as also was observed for ALD (atomic layer deposition) prepared $\mathrm{Sr}$ doped $\mathrm{HfO}_{2}$ by Schenk et al. ${ }^{38}$ After $10 \mathrm{k}$ cycles, a remanent polarization of $10 \mu \mathrm{C} / \mathrm{cm}^{2}$ is reached. By applying a quasistatic positive voltage sweep with a series resistor of $10 \mathrm{k} \Omega$ to prevent the device from complete breakdown, an electroforming step is carried out as depicted in the inset of Fig. 1(c). After the forming step into the on state and the first reset into the off state, a reproducible resistive VCM switching can be observed, as shown in Fig. 1(d).

In the following, the wake-up for both layer configurations induced by single and double pulses is investigated. Therefore, four different pulse patterns are applied in each case to a pristine device: (a) one positive pulse, (b) one negative pulse, (c) a negative pulse followed by a positive pulse and (d) a positive pulse followed by a negative pulse. An electrical field of $3 \mathrm{MV} / \mathrm{cm}$ is applied for all pulse patterns, whereby the pulse length for every single pulse is varied between $0.5 \mathrm{~s}$ and $10 \mathrm{~s}$. The resulting $\mathrm{I}(\mathrm{V})$ curves measured after the pulse wake-up for the symmetrical devices are
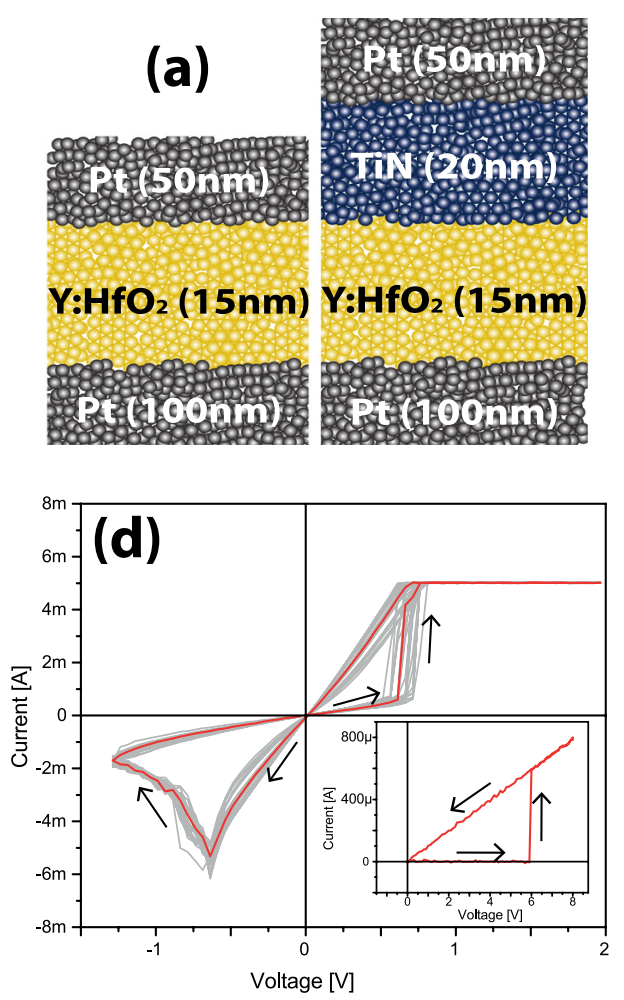

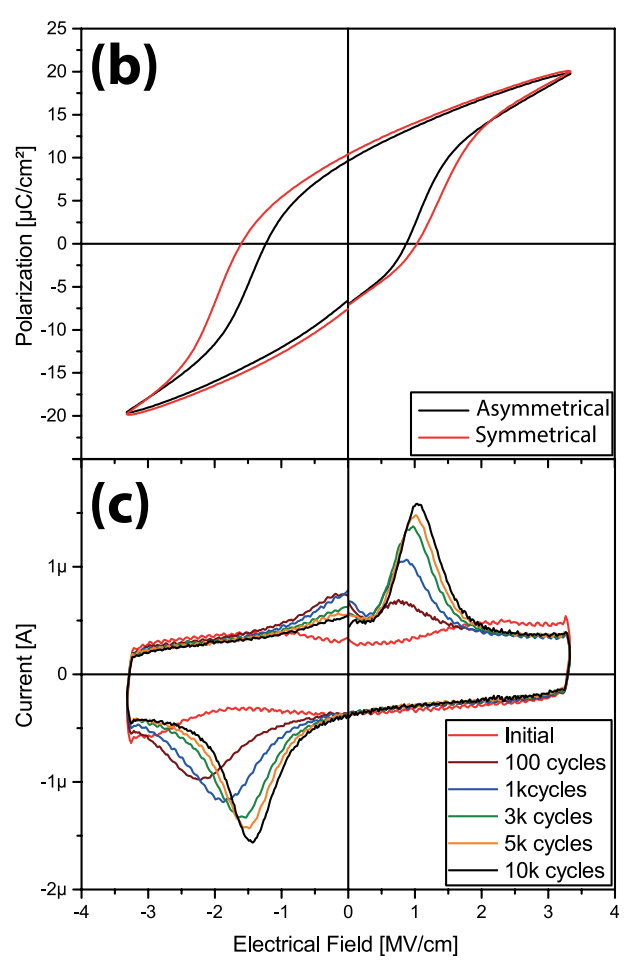

FIG. 1. (a) Two devices used in this work, which differ by an additional TiN layer. (b) Hysteresis curves after wake-up for the symmetrical and asymmetrical stack. (c) Current curves for a regular wake-up at a frequency of $1 \mathrm{kHz}$ for the asymmetrical stack. (d) Resulting resistive switching curves after a forming step is realized with a $10 \mathrm{k} \Omega$ series resistor (inset). 

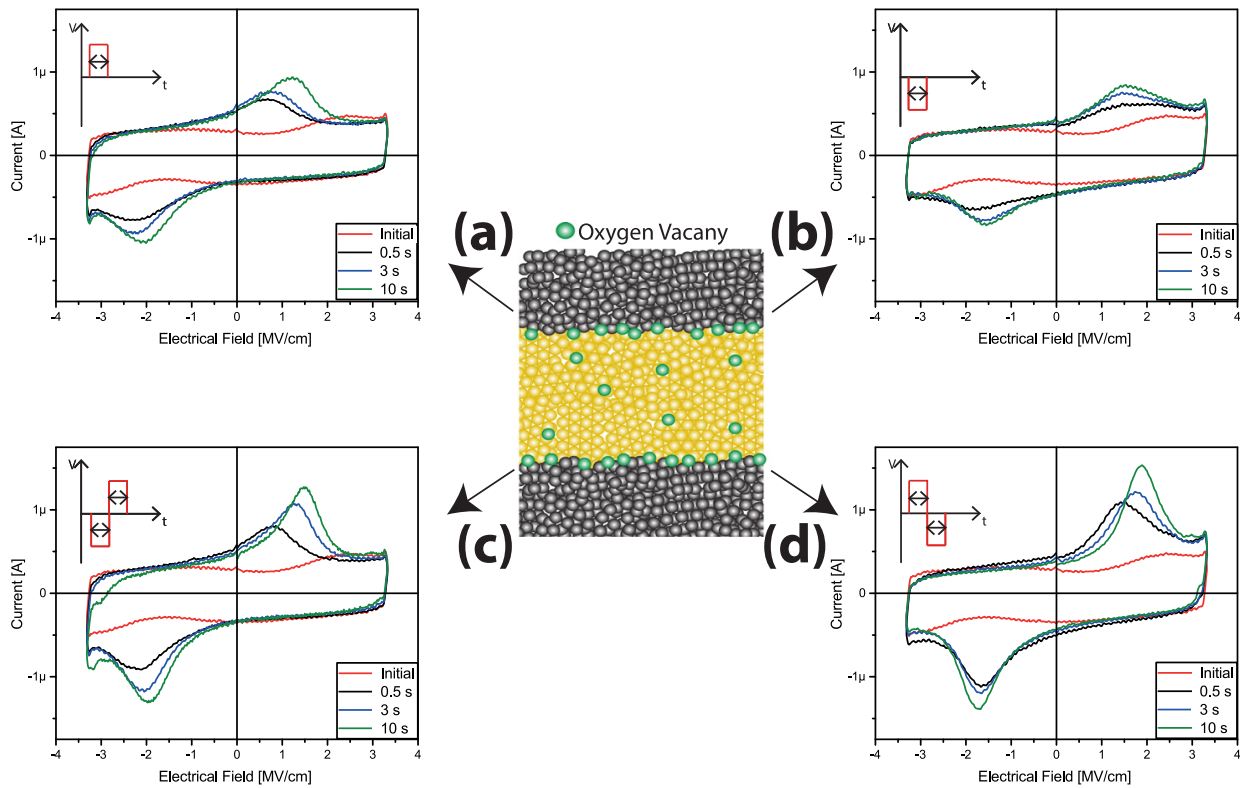

FIG. 2. Resulting IV curves for the symmetrical device at varying pulse lengths and different pulse pattern, which are depicted in the inset of each graph. Each pulse pattern and time was applied on a virgin device, respectively. An electrical field of $3 \mathrm{MV} / \mathrm{cm}$ was used for all pulses. shown in Fig. 2. In the middle, the assumed distribution of oxygen vacancies before an applied voltage is shown. Due to the symmetrical electrodes, an almost symmetrical distribution of oxygen vacancies is expected (small deviations can be given due to the different treatments of the both electrodes during deposition of the layers, which is described in detail in Ref. 36). Hence, the most vacancies are accumulated at the both interfaces between the hafnium oxide and the platinum electrode, as also assumed by Pešic et al. ${ }^{19}$ Such a distribution of oxygen vacancies does lead to an internal bias field with the characteristic double current peaks, as described by Schenk et al. ${ }^{16}$ For the two single pulses ((a) and (b)), a small wake-up can be measured indicated by the increasing current peak with an increasing pulse length. Thus, the oxygen vacancies drift to the bottom and top electrode, respectively. This redistribution leads to a phase transition to the ferroelectric orthorhombic phase. ${ }^{19}$ Both polarities of the single pulse wake-up show almost identical results, whereby the current peaks increase for increasing pulse times. For the two double pulses ((c) and (d)), a more distinct current peak can be found compared to the single pulses. For increasing pulse lengths, not only the current peak increases but also the I(V) curves get more symmetrical. Due to the two pulses, most likely, a more homogeneous
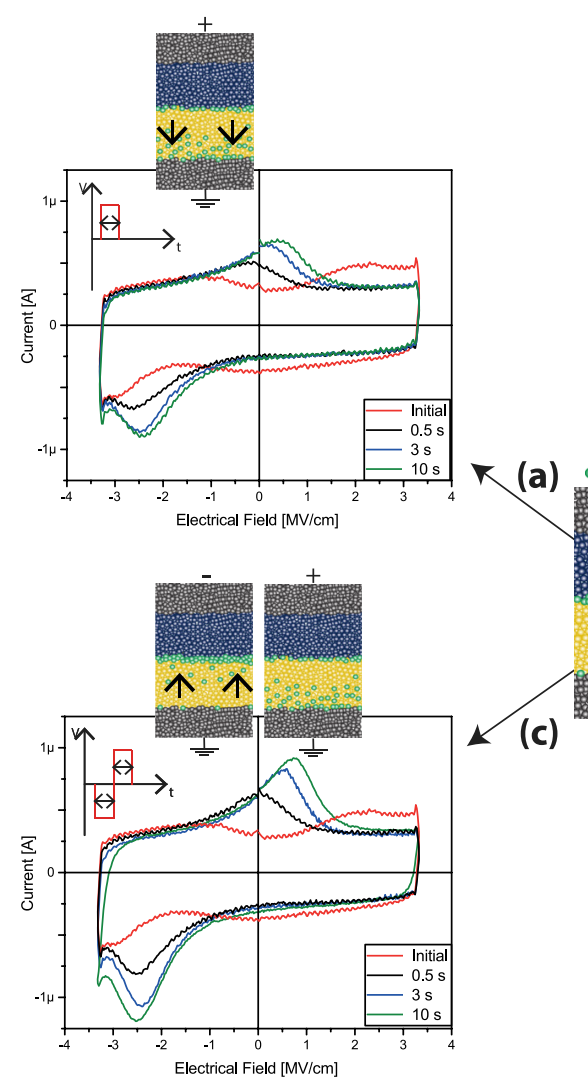

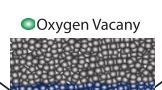

(b)
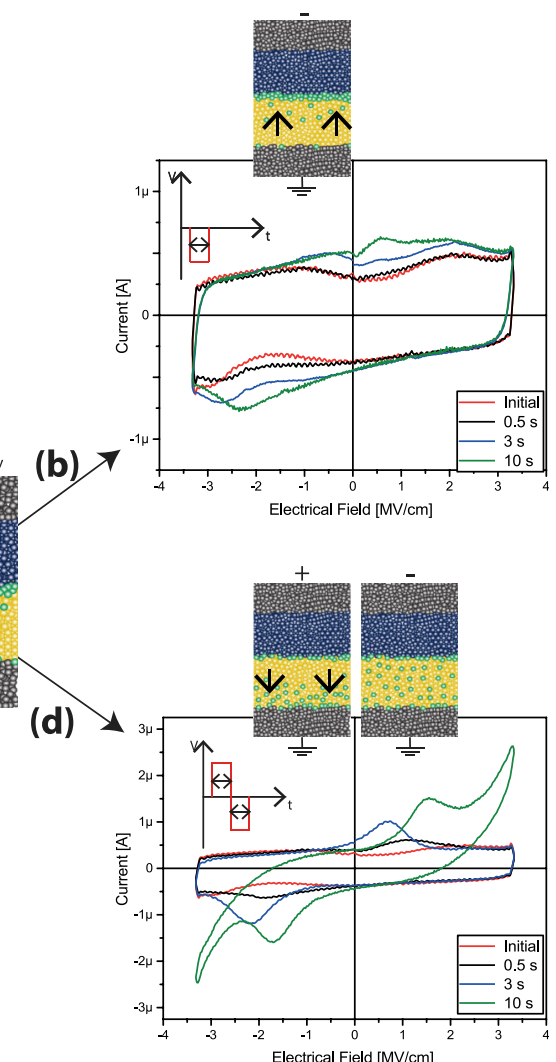

FIG. 3. Resulting IV curves for the asymmetrical device at varying pulse lengths and different pulse pattern, which are depicted in the inset of each graph. Above each graph, the assumed oxygen vacancies distribution is shown. Each pulse pattern and time was applied on a virgin device, respectively. An electrical field of $3 \mathrm{MV} / \mathrm{cm}$ was used for all pulses. 
distribution of the oxygen vacancies is achieved, leading to a larger amount of ferroelectric grains.

For the asymmetrical device with the TiN top electrode, the distribution of the oxygen vacancies is expected to be strongly different. The assumed initial distribution is shown in the middle of Fig. 3. Most of the oxygen vacancies are accumulated at the top interface due to oxidation of the TiN at the $\mathrm{TiN} / \mathrm{HfO}_{2}$ interface, as also reported elsewhere. ${ }^{19,39-41}$ The same pulse patterns as well as the same pulse lengths have been applied to the asymmetrical layer stack, as shown before for the symmetrical stack. For the positive single pulse (Fig. 3(a)), a wake-up is observed for all applied pulse lengths. The asymmetrical current peaks shifted towards negative voltages indicate a strong imprint.

This imprint can be explained by the strong accumulation of the positively charged oxygen vacancies at the top interface, whereby a positive built in bias voltage results. During the applied pulse, some oxygen vacancies drift from the top to the bottom leading to a partially formation of the ferroelectric phase. For longer pulse lengths, more vacancies are activated and therefore a stronger wake-up can be observed. For the negative single pulse (Fig. 3(b)), almost no wake-up can be observed. The negative voltage applied to the top electrode is partially compensated by the voltage bias induced by the vacancies distribution. Furthermore, most of the positively charged vacancies are accumulated at the top interface and therefore a negative pulse does only lead to a minor redistribution of oxygen vacancies within the hafnium oxide layer. The applied double pulse with a negative pulse followed by a positive one (Fig. 3(c)) shows similar results compared to the single positive pulse. The resulting current peaks are indeed slightly higher, but for both pulse patterns a strong imprint is found for all pulse durations. The first negative pulse has only a small influence on the vacancy distribution; therefore, the wake-up is dominated by the following positive pulse. This does lead to a final vacancy distribution as for the single positive pulse (Fig. 3(a)). For the double pulses where first a positive pulse followed by a negative pulse is applied (Fig. 3(d)), a lower imprint can be observed compared to the pattern (c). This can be understood by the order of the two pulses. The first positive pulse does lead to a drift of the oxygen vacancies from the top TiN interface towards the bottom interface. Due to the changed distribution, the following negative pulse induces a more homogenous distribution of oxygen vacancies within the layer and therefore a lower imprint compared to the (c) pattern is achieved. A further significant difference can be found for the $10 \mathrm{~s}$ pulses. On the one hand, the current peaks are symmetrical; therefore, no internal bias is left, e.g., a symmetrical oxygen vacancy distribution is established. On the other hand, the leakage currents strongly increase, which can be attributed to the generation of new oxygen vacancies. The generation of oxygen vacancies as the origin of the increasing leakage current was also assumed by Pešic et al. for the increasing cycling numbers. ${ }^{19}$ The difference of the amount of oxygen vacancies between the top and bottom interface can be estimated by the internal bias field, as shown by Park et $a l{ }^{42}$ Therefore, the shift of the current peaks for the $3 \mathrm{~s}$ pulse duration of the pulse pattern (c) (negative pulse followed by a positive pulse) is exemplary used to examine the amount of oxygen vacancies. For the symmetrical stack, an internal bias of $-0.67 \mathrm{MV} / \mathrm{cm}$ is observed, whereas for the asymmetrical stack an internal bias of $-1.25 \mathrm{MV} / \mathrm{cm}$ is measured. This indicates that for both stacks a larger amount of oxygen vacancies is accumulated at the top interface compared to the bottom interface. The concentration of charged defects (n) can be simply estimated by using the following equation:

$$
E_{\text {bias }}=\frac{n q}{\varepsilon_{0} \varepsilon_{r}},
$$

where $q$ is the charge of the defects, $\varepsilon_{0}$ the vacuum permittivity, and $\varepsilon_{r}$ the relative permittivity of the dielectric film. This results in a larger amount of oxygen vacancies at the top interface of $0.56 \times 10^{13} \mathrm{~cm}^{-2}$ and $1.04 \times 10^{13} \mathrm{~cm}^{-2}$ for the symmetrical and asymmetrical stack, respectively. These values are slightly larger as those calculated by Park et al., which is due to the larger bias field. ${ }^{42}$ This amount of vacancies is small compared to the overall amount of vacancies expected
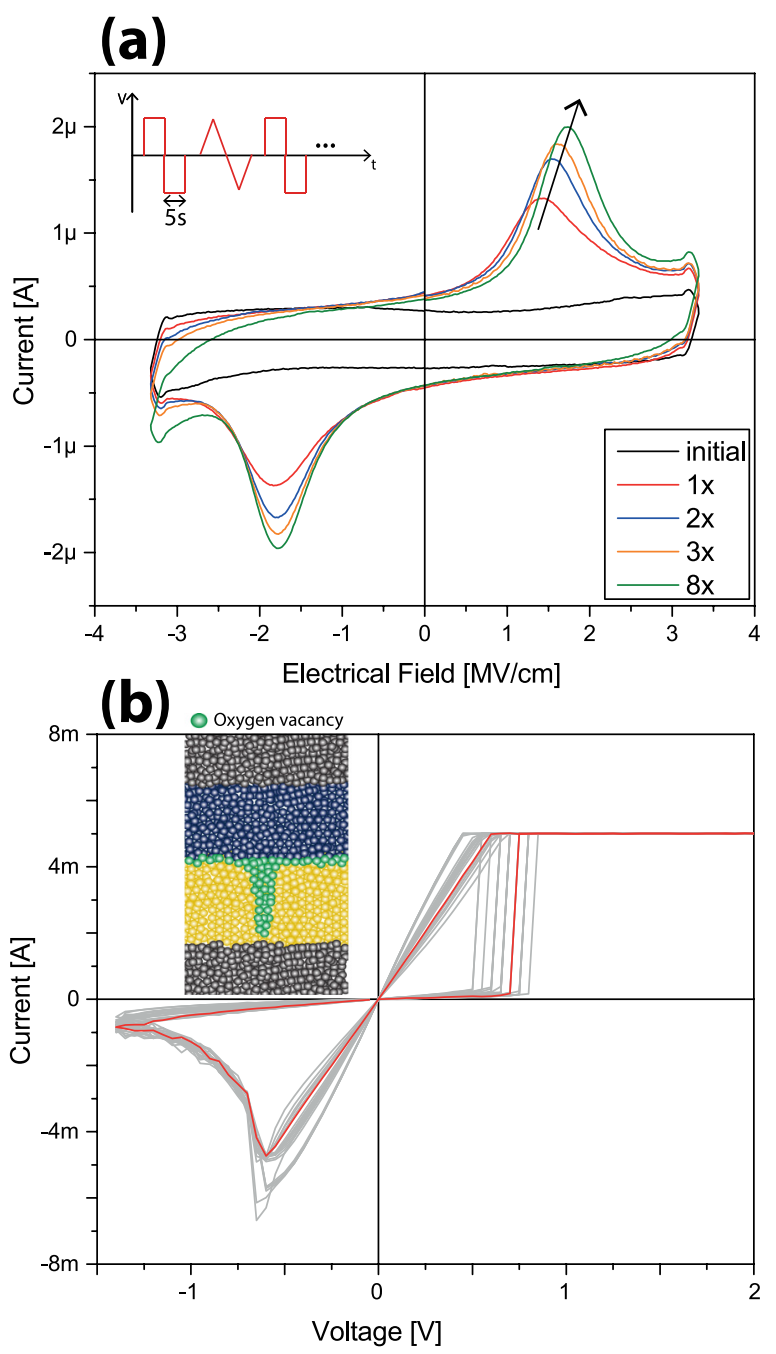

FIG. 4. (a) Resulting current curves for repeating double wake-up pulses with a length of $5 \mathrm{~s}$ for the asymmetrical layer stack. During the 9 th cycle, an electrical breakdown occurs. A series resistance of $10 \mathrm{k} \Omega$ is used to prevent the device from a full break down. (b) Resistive switching VCM curves after the breakdown. The inset shows the conductive path formed by oxygen vacancies. 
due to the 5\% doping with the trivalent yttrium, which should result in an overall vacancy concentration of $2.5 \%$.

Another factor that can influence the built in bias is the difference in the work functions of TiN and Pt. Assuming a linear internal potential over the hafnium oxide and work functions of $5.6 \mathrm{eV}$ and $4.8 \mathrm{eV}$ for platinum and titanium nitride, respectively, results in a negative bias of $-0.53 \mathrm{MV} / \mathrm{cm}$. Nevertheless, this is a very simplified assumption, because due to the high doping concentration within the hafnia film no linear behavior of the potential is expected. In addition, with further cycling as shown in Fig. 4(a), symmetric coercitive fields are reached, which also disagrees with the assumption of a built in bias due to the different electrodes.

Repeating the double pulse pattern does lead to a stronger wake up, as shown exemplary for the (d) pulse pattern in Fig. 4(a). Furthermore, the leakage current increases which manifests in particular at the negative and positive maximum voltages. During the 9th cycle, a breakdown occurs, whereupon a reproducible resistive VCM switching is observed, as depicted in Fig. 4(b). That means that during the wake-up oxygen vacancies are generated and at a certain point the electroforming process sets in and the vacancies are accumulated to form a conductive path through the oxide layer (see the inset of Fig. 4(b)). ${ }^{43}$ Once the conductive path is formed, the device is shortened and therefore no ferroelectric switching can be observed any more. Pešic et al. showed that the leakage current is dominated by the oxygen vacancies at the grain boundaries, and therefore, it is assumed that the generated vacancies are accumulated at a grain boundary where the formation of the filament also takes place. ${ }^{19,28,44}$

In the next step, the frequency dependence of the dielectric breakdown is investigated for the symmetrical and asymmetrical devices. For each frequency and layer stack, ten devices were measured to obtain the device-to-device variation. Before the breakdown measurements, a wake-up step was performed with $1 \mathrm{~K}$ cycles and $10 \mathrm{~K}$ cycles at $1 \mathrm{kHz}$ for the symmetrical and asymmetrical devices, respectively. Fig. 5(a) shows the number of cycles which could be performed till the dielectric breakdown in dependence of the cycling frequency in the range between $10 \mathrm{kHz}$ and $100 \mathrm{kHz}$. The red circles indicate the asymmetrical device where no breakdown was observed after the performed cycling. For the symmetrical devices with inert platinum electrodes, a much larger number of cycles could be performed until breakdown compared to the asymmetrical devices especially at the lower frequencies. Furthermore, for both layer stacks, an exponential dependence of the performed cycles in dependence of cycling frequency is observed. An increase of the cycling frequency by a factor of 10 for example leads to an increase of the cycles-until-breakdown by a factor of 1000 for the asymmetrical devices. For higher frequencies, a much larger scattering is also observed compared to lower frequencies for the asymmetrical devices. Fig. 5(b) shows exemplary curves of the remanent polarization in dependence of the number of cycles for different frequencies for the asymmetrical device. It is known for conventional ferroelectric as $\mathrm{PZT}^{45,46}$ as well as for hafnia based ferroelectrics ${ }^{2,10,47}$ that the remanent polarization is reduced during cycling which is known as the fatigue-effect. For our devices, we observe an additional effect. For lower frequencies, the remanent polarization increases with cycling, which is not expected for fatigue measurements, whereas for larger frequencies a reduction of the remanent polarization is observed. The hysteresis and current curves after wake-up and after cycling are shown exemplary for $30 \mathrm{kHz}$ and $100 \mathrm{kHz}$ in Fig. 5(c). For both frequencies, the switching current peaks are reduced
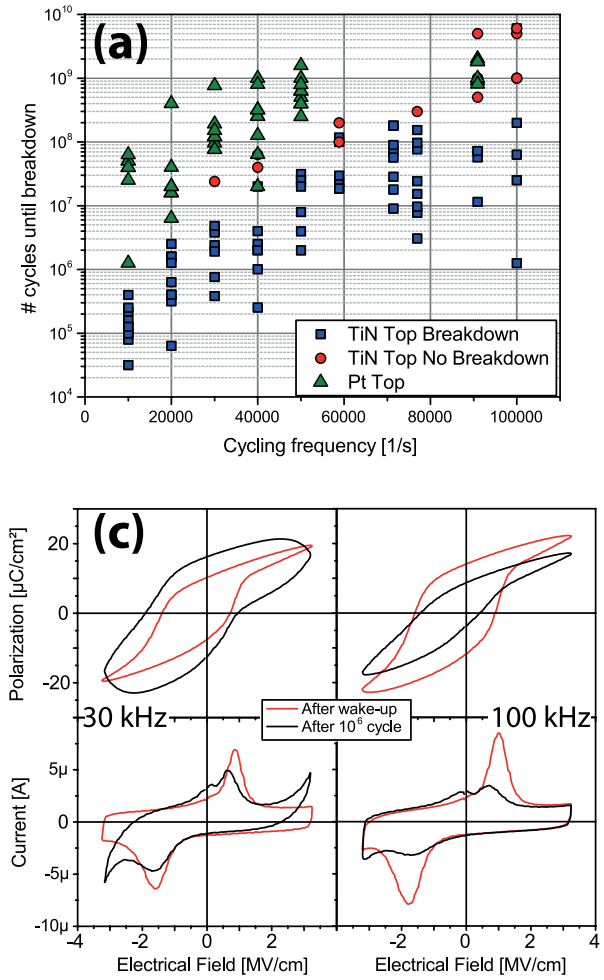
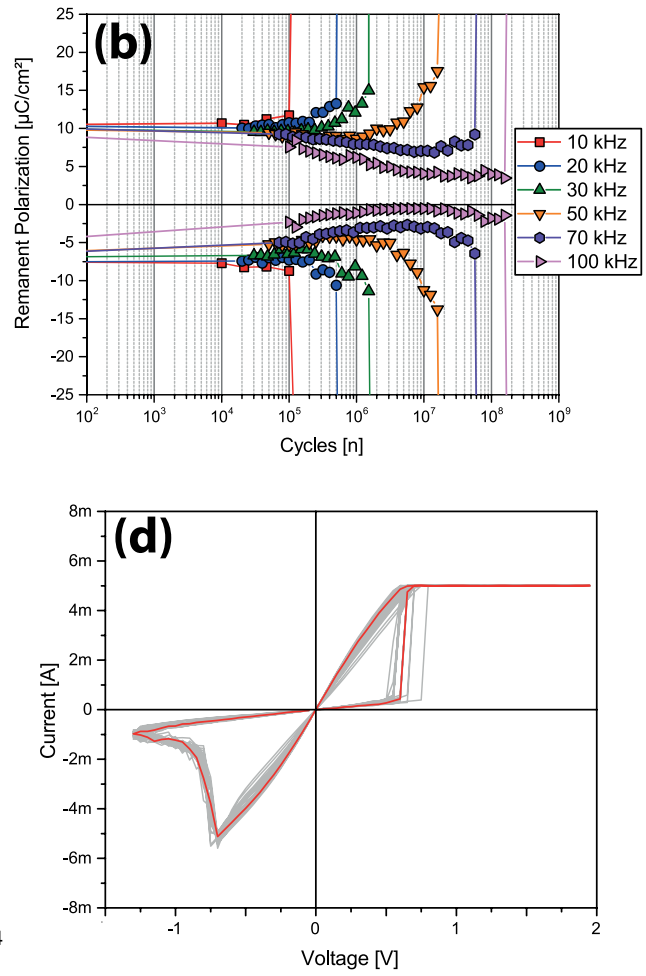

FIG. 5. (a) Number of cycles that can be performed on the symmetrical and asymmetrical devices till a breakdown occurs in dependence of the cycling frequency. The red circles indicate the devices where no breakdown was reached after the amount of cycles. (b) Exemplary fatigue results for the asymmetrical stack for different frequencies showing the evolution of the remanent polarization over the number of cycles. (c) $\mathrm{I}(\mathrm{V})$ and $\mathrm{P}(\mathrm{V})$ curves for a cycling frequency of $30 \mathrm{kHz}$ and $100 \mathrm{kHz}$ after wake-up and after $10^{6}$ cycles, respectively. (d) Resulting resistive VCM switching curves after a breakdown reached with a fatigue frequency of $100 \mathrm{kHz}$. A $10 \mathrm{k} \Omega$ series resistance was used during breakdown to prevent the device from a complete breakdown. 
after cycling, indicating a reduction of the ferroelectric response. After cycling at $30 \mathrm{kHz}$, the leakage current strongly increases, which explains the measured increase in remanent polarization. ${ }^{48}$ Since it is assumed that the leakage current is dominated by the amount of oxygen vacancies within the oxide layer, as also shown by Pešic et al. ${ }^{19}$ it seems that for higher frequencies the generation of oxygen vacancies is strongly suppressed, whereby the endurance of the devices increases. By using a series resistance during the breakdown measurements, as for the sweep forming (Fig. 1(c)) and the long pulses forming (Fig. 4(b)), a reproducible resistive VCM switching is found after the breakdown at $100 \mathrm{kHz}$ (Fig. 5(d)). These results show that by applying a voltage to the device not only ferroelectric switching but also movement and generation of oxygen vacancies takes place. Controlling these processes is crucial to achieve a high endurance for the ferroelectric switching. A further interesting observation is that the resistive switching curves for the different forming steps look very similar although the forming conditions are strongly different using a quasi static sweep at one polarity (Fig. 1(c)), a pulse sequence at $0.1 \mathrm{~Hz}$ (Fig. 4(b)) and a forming at a high frequency of $100 \mathrm{kHz}$ (Fig. 5(d)). Therefore, it seems that the applied forming conditions do not strongly influence the resulting filament.

\section{CONCLUSIONS}

In summary, we have shown that a wake-up in thin yttrium doped hafnium oxide can be realized by single voltage pulses. Furthermore, from the pulse measurements, the initial distribution of oxygen vacancies was extracted showing that for an asymmetrical device oxygen vacancies are accumulated at the TiN interface leading to an internal bias field. It was shown that the degradation of the device involves the generation of oxygen vacancies leading to a dielectric breakdown. The generation of oxygen vacancies shows a strong frequency dependence, whereby at larger frequencies the generation is suppressed leading to a strongly increased lifetime of the device. The shown resistive VCM switching is a failure mechanism in ferroelectric thin layers, which needs to be investigated in more detail to increase the lifetime of future ferroelectric memory devices.

\section{ACKNOWLEDGMENTS}

This work was funded by the German Research Foundation (Deutsche Forschungsgemeinschaft) within the scope of the project "Inferox" (Project No. BO 1629/10-2).

${ }^{1}$ T. S. Boescke, J. Mueller, D. Braeuhaus, U. Schroeder, and U. Boettger, "Ferroelectricity in hafnium oxide thin films," Appl. Phys. Lett. 99, 102903 (2011).

${ }^{2}$ S. Mueller, J. Mueller, U. Schroeder, and T. Mikolajick, "Reliability characteristics of ferroelectric $\mathrm{Si}: \mathrm{HfO}_{2}$ thin films for memory applications," IEEE Trans. Device Mater. Reliab. 13, 93-97 (2013).

${ }^{3}$ T. Mikolajick, S. Müller, T. Schenk, E. Yurchuk, S. Slesazeck, U. Schröder, S. Flachowsky, R. van Bentum, S. Kolodinski, P. Polakowski, and J. Müller, "Doped hafnium oxide-An enabler for ferroelectric field effect transistors," Adv. Sci. Technol. 95, 136-145 (2014).

${ }^{4}$ J. Mueller, P. Polakowski, S. Mueller, and T. Mikolajick, "Ferroelectric hafnium oxide based materials and devices: Assessment of current status and future prospects," ECS J. Solid State Sci. Technol 4, N30-N35 (2015).

${ }^{5}$ X. Sang, E. D. Grimley, T. Schenk, U. Schroeder, and J. M. LeBeau, "On the structural origins of ferroelectricity in $\mathrm{HfO}_{2}$ thin films," Appl. Phys. Lett. 106, 162905 (2015).

${ }^{6}$ D. Martin, J. Mueller, T. Schenk, T. M. Arruda, A. Kumar, E. Strelcov, E. Yurchuk, S. Mueller, D. Pohl, U. Schroeder, S. V. Kalinin, and T. Mikolajick, "Ferroelectricity in Si-Doped $\mathrm{HfO}_{2}$ revealed: A binary leadfree ferroelectric," Adv. Mater. 26, 8198-8202 (2014).

${ }^{7}$ R. Materlik, C. Künneth, and A. Kersch, "The origin of ferroelectricity in $\mathrm{Hf}_{1-\mathrm{x}} \mathrm{Zr}_{\mathrm{x}} \mathrm{O}_{2}$ : A computational investigation and a surface energy model," J. Appl. Phys. 117, 134109 (2015).

${ }^{8}$ D. Zhou, J. Xu, Q. Li, Y. Guan, F. Cao, X. Dong, J. Mueller, T. Schenk, and U. Schroeder, "Wake-up effects in Si-doped hafnium oxide ferroelectric thin films," Appl. Phys. Lett. 103, 192904 (2013).

${ }^{9}$ S. Mueller, C. Adelmann, A. Singh, S. Van Elshocht, U. Schroeder, and T. Mikolajick, "Ferroelectricity in Gd-Doped $\mathrm{HfO}_{2}$ thin films," ECS J. Solid State Sci. Technol. 1, N123-N126 (2012).

${ }^{10}$ T. Schenk, S. Mueller, U. Schroeder, R. Materlik, A. Kersch, M. Popovici, C. Adelmann, S. Van Elshocht, and T. Mikolajick, "Strontium doped hafnium oxide thin films: Wide process window for ferroelectric memories," in Proceedings of the ESSDERC (2013).

${ }^{11}$ M. Hyuk Park, H. Joon Kim, Y. Jin Kim, Y. Hwan Lee, T. Moon, K. Do Kim, S. Dam Hyun, F. Fengler, U. Schroeder, and C. Seong Hwang, "Effect of $\mathrm{Zr}$ content on the wake-up effect in $\mathrm{Hf}_{1-\mathrm{x}} \mathrm{Zr}_{\mathrm{x}} \mathrm{O}_{2}$ films," ACS Appl. Mater. Interfaces 8(24), 15466-15475 (2016).

${ }^{12}$ T. Olsen, U. Schroeder, S. Mueller, A. Krause, D. Martin, A. Singh, J. Mueller, M. Geidel, and T. Mikolajick, "Co-sputtering yttrium into hafnium oxide thin films to produce ferroelectric properties," Appl. Phys. Lett. 101, 82905 (2012).

${ }^{13}$ S. Starschich, D. Griesche, T. Schneller, R. Waser, and U. Boettger, "Chemical solution deposition of ferroelectric yttrium-doped hafnium oxide films on platinum electrodes," Appl. Phys. Lett. 104, 202903 (2014).

${ }^{14}$ T. Shimizu, K. Katayama, T. Kiguchi, A. Akama, T. J. Konno, O. Sakata, and H. Funakubo, "The demonstration of significant ferroelectricity in epitaxial Y-doped $\mathrm{HfO}_{2}$ film,” Sci. Rep. 6, 32931 (2016).

${ }^{15}$ M. Hyuk Park, H. Joon Kim, Y. Jin Kim, Y. Hwan Lee, T. Moon, K. Do Kim, S. Dam Hyun, and C. Seong Hwang, "Study on the size effect in $\mathrm{Hf}_{0.5} \mathrm{Zr}_{0.5} \mathrm{O}_{2}$ films thinner than $8 \mathrm{~nm}$ before and after wake-up field cycling," Appl. Phys. Lett. 107, 192907 (2015).

${ }^{16}$ T. Schenk, M. Hoffmann, J. Ocker, M. Pešic, T. Mikolajick, and U. Schroeder, "Complex internal bias fields in ferroelectric hafnium oxide," ACS Appl. Mater. Interfaces 7, 20224-20233 (2015).

${ }^{17}$ P. D. Lomenzo, Q. Takmeel, C. Zhou, C. M. Fancher, E. Lambers, N. G. Rudawski, J. L. Jones, S. Moghaddam, and T. Nishida, "TaN interface properties and electric field cycling effects on ferroelectric Si-doped $\mathrm{HfO}_{2}$ thin films," J. Appl. Phys. 117, 134105 (2015).

${ }^{18}$ S. Starschich, S. Menzel, and U. Böttger, "Evidence for oxygen vacancies movement during wake-up in ferroelectric hafnium oxide," Appl. Phys. Lett. 108, 032903 (2016).

${ }^{19}$ M. Pešic, F. P. G. Fengler, L. Larcher, A. Padovani, T. Schenk, E. D. Grimley, X. Sang, J. M. LeBeau, S. Slesazeck, U. Schroeder, and T. Mikolajick, "Physical mechanisms behind the field-cycling behavior of HfO2-based ferroelectric capacitors," Adv. Funct. Mater. 26, 4601-4612 (2016).

${ }^{20}$ E. D. Grimley, T. Schenk, X. Sang, M. Pešic', U. Schroeder, T. Mikolajick, and J. M. LeBeau, "Structural changes underlying fieldcycling phenomena in ferroelectric $\mathrm{HfO}_{2}$ thin films," Adv. Electron. Mater. 2, 1600173 (2016).

${ }^{21}$ F. P. G. Fengler, M. Pešic', S. Starschich, T. Schneller, C. Künneth, U. Böttger, H. Mulaosmanovic, T. Schenk, M. H. Park, R. Nigon, P. Muralt, T. Mikolajick, and U. Schroeder, "Domain pinning: Comparison of hafnia and PZT based ferroelectrics," Adv. Elekectron. Mater. 3, 1600505 (2017).

${ }^{22}$ S. Brivio, J. Frascaroli, and S. Spiga, "Role of metal-oxide interfaces in the multiple resistance switching regimes of $\mathrm{Pt} / \mathrm{HfO} 2 / \mathrm{TiN}$ devices," Appl. Phys. Lett. 107, 23504 (2015).

${ }^{23}$ H. Y. Lee, P. S. Chen, T. Y. Wu, Y. S. Chen, C. C. Wang, P. J. Tzeng, C. H. Lin, F. Chen, C. H. Lien, and M.-J. Tsai, "Low power and high speed bipolar switching with a thin reactive Ti buffer layer in robust $\mathrm{HfO}_{2}$ based RRAM," 2008 IEEE International Electron Devices Meeting, San Francisco, CA, 2008, pp. 1-4.

${ }^{24}$ M. Sowinska, T. Bertaud, D. Walczyk, S. Thiess, P. Calka, L. Alff, C. Walczyk, and T. Schroeder, "In-operando hard X-ray photoelectron spectroscopy study on the impact of current compliance and switching cycles 
on oxygen and carbon defects in resistive switching Ti/HfO2/TiN cells," J. Appl. Phys. 115, 204509 (2014).

${ }^{25}$ L. Zhao, H. Chen, S. Wu, Z. Jiang, S. Yu, T. Hou, H. P. Wong, and Y. Nishi, "Multi-level control of conductive nano-filament evolution in $\mathrm{HfO} 2$ ReRAM by pulse-train operations," Nanoscale 6, 5698-5702 (2014).

${ }^{26}$ L. Goux, X. P. Wang, Y. Y. Chen, L. Pantisano, N. Jossart, B. Govoreanu, J. A. Kittl, M. Jurczak, L. Altimime, and D. J. Wouters, "Roles and effects of TiN and Pt electrodes in resistive-switching HfO2 systems," Electrochem. Solid-State Lett. 14, H244 (2011).

${ }^{27}$ P. Gonon, M. Mougenot, C. Vallee, C. Jorel, V. Jousseaume, H. Grampeix, and F. El Kamel, "Resistance switching in HfO2 metal-insulator-metal devices," J. Appl. Phys. 107, 74507 (2010).

${ }^{28}$ M. Lanza, G. Bersuker, M. Porti, E. Miranda, M. Nafria, and X. Aymerich, "Resistive switching in hafnium dioxide layers: Local phenomenon at grain boundaries," Appl. Phys. Lett. 101, 193502 (2012).

${ }^{29}$ J. J. Yang, M. D. Pickett, X. Li, D. A. A. Ohlberg, D. R. Stewart, and R. S. Williams, "Memristive switching mechanism for metal/oxide/metal nanodevices," Nat. Nanotechnol. 3, 429-433 (2008).

${ }^{30}$ S. Larentis, F. Nardi, S. Balatti, D. C. Gilmer, and D. Ielmini, "Resistive switching by voltage-driven ion migration in bipolar RRAM-Part II: Modeling," IEEE Trans. Electron Devices 59, 2468-2475 (2012).

${ }^{31}$ A. Marchewka, B. Roesgen, K. Skaja, H. Du, C. L. Jia, J. Mayer, V. Rana, R. Waser, and S. Menzel, "Nanoionic resistive switching memories: On the physical nature of the dynamic reset process," Adv. Electron. Mater. 2, 1500233 (2016).

${ }^{32}$ R. Waser, R. Dittmann, G. Staikov, and K. Szot, "Redox-based resistive switching memories - nanoionic mechanisms, prospects, and challenges," Adv. Mater. 21, 2632-2663 (2009).

${ }^{33}$ J. J. Yang, F. Miao, M. D. Pickett, D. A. A. Ohlberg, D. R. Stewart, C. N. Lau, and R. S. Williams, "The mechanism of electroforming of metal oxide memristive switches," Nanotechnology 20, 215201 (2009).

${ }^{34}$ E. Abbaspour, S. Menzel, and C. Jungemann, "The role of the interface reactions in the electroforming of redox-based resistive switching devices using KMC simulations," in Proceedings of the International Conference On Simulation of Semiconductor Processes and Devices (SISPAD) (Washington DC, USA, 2015), pp. 293-296.

${ }^{35}$ S. U. Sharath, T. Bertaud, J. Kurian, E. Hildebrandt, C. Walczyk, P. Calka, P. Zaumseil, M. Sowinska, D. Walczyk, A. Gloskovskii, T. Schroeder, and L. Alff, "Towards forming-free resistive switching in oxygen engineered HfO2-x," Appl. Phys. Lett. 104, 063502 (2014).

${ }^{36} \mathrm{~S}$. Starschich, D. Griesche, T. Schneller, and U. Böttger, "Chemical solution deposition of ferroelectric hafnium oxide for future lead free ferroelectric devices," ECS J. Solid State Sci. Technol. 4, P419-P423 (2015).
${ }^{37}$ S. Starschich and U. Boettger, "An extensive study of the influence of dopants on the ferroelectric properties of $\mathrm{HfO}_{2}$," J. Mater. Chem. C 5, 333-338 (2017).

${ }^{38}$ T. Schenk, U. Schroeder, M. Pešic, M. Popovici, Y. V. Pershin, and T. Mikolajick, "Electric field cycling behavior of ferroelectric hafnium oxide,” ACS Appl. Mater. Interfaces 6, 19744-19751 (2014).

${ }^{39}$ M. H. Park, H. J. Kim, Y. J. Kim, T. Moon, K. D. Kim, and C. S. Hwang, "Thin $\mathrm{Hf}_{\mathrm{x}} \mathrm{Zr}_{1-\mathrm{x}} \mathrm{O}_{2}$ films: A new lead-free system for electrostatic supercapacitors with large energy storage density and robust thermal stability," Adv. Energy Mater. 4, 1400610 (2014).

${ }^{40}$ M. Hyuk Park, H. Joon Kim, Y. Jin Kim, W. Jeon, T. Moon, and C. Seong Hwang, "Ferroelectric properties and switching endurance of $\mathrm{Hf}_{0.5} \mathrm{Zr}_{0.5} \mathrm{O}_{2}$ films on TiN bottom and $\mathrm{TiN}$ or $\mathrm{RuO}_{2}$ top electrodes," Phys. Status Solidi RRL 8, 532-535 (2014).

${ }^{41}$ S. Yu, H.-Y. Chen, B. Gao, J. Kang, and H.-S. P. Wong, "HfOx-based vertical resistive switching random access memory suitable for bit-cost-effective three-dimensional cross-point architecture," ACS Nano 7, 2320-2325 (2013).

${ }^{42}$ M. H. Park, H. J. Kim, Y. J. Kim, T. Moon, K. D. Kim, Y. H. Lee, S. D. Hyun, and C. S. Hwang, "Study on the internal field and conduction mechanism of atomic layer deposited ferroelectric $\mathrm{Hf}_{0.5} \mathrm{Zr}_{0.5} \mathrm{O}_{2}$ thin films," J. Mater. Chem. C 3, 6291-6300 (2015).

${ }^{43}$ E. Yalon, I. Karpov, V. Karpov, I. Riess, D. Kalaev, and D. Ritter, "Detection of the insulating gap and conductive filament growth direction in resistive memories," Nanoscale 7, 15434-15441 (2015).

${ }^{44}$ G. Bersuker, J. Yum, L. Vandelli, A. Padovani, L. Larcher, V. Iglesias, M. Porti, M. Nafria, K. McKenna, A. Shluger, P. Kirsch, and R. Jammy, "Grain boundary-driven leakage path formation in $\mathrm{HfO} 2$ dielectrics," Solid-State Electron. 65-66, 146-150 (2011).

${ }^{45}$ J. S. Cross, S. Kim, S. Wada, and A. Chatterjee, "Characterization of Bi and Fe co-doped PZT capacitors for FeRAM," Sci. Technol. Adv. Mater. 11, 044402 (2010).

${ }^{46}$ D. Braeuhaus, P. J. Schorn, U. Boettger, and R. Waser, "Signal form influences on the fatigue behavior of PZT thin film capacitors," in Proceedings of the 16th IEEE International Symposium on Applications of Ferroelectrics, 27-31 May 2007 (IEEE, Nara, Japan 2007), pp. 69-71.

${ }^{47}$ U. Schroeder, S. Mueller, J. Mueller, E. Yurchuk, D. Martin, C. Adelmann, T. Schloesser, R. van Bentum, and T. Mikolajick, "Hafnium oxide based CMOS compatible ferroelectric materials," ECS J. Solid State Sci. Technol. 2, N69-N72 (2013).

${ }^{48}$ T. Schenk, E. Yurchuk, S. Mueller, U. Schroeder, S. Starschich, U. Bottger, and T. Mikolajick, "About the deformation of ferroelectric hystereses,” Appl. Phys. Rev. 1, 041103 (2014). 